\title{
From predisposition to illness: genetically sensitive intermediate pathways to mood disorders $^{\dagger}$
}

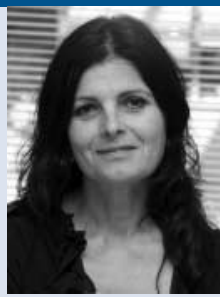

Anne Duffy

\section{Summary}

The contemporary model of mood disorders proposes that multiple susceptibility genes interact with multiple other risk factors. However, the specific nature of the genetic vulnerability and the intermediate causal pathways are not known. In this edition of the Journal, Goodyer and colleagues report new findings suggesting genetic moderation of an association between elevated cortisol and depression in high-risk adolescents.

\section{Declaration of interest}

None.
Anne Duffy is a Professor and Senior Clinical Research Scholar in the Department of Psychiatry at Dalhousie University in Halifax, Nova Scotia. She is funded by a grant from the Canadian Institutes of Health Research to study the early clinical stages and associated neurobiological correlates of bipolar disorder in high-risk offspring.

\section{The current model of primary mood disorders}

One could argue that over the past 25 years not much has changed in regard to the common conceptualisation of mood disorders; that is, multiple susceptibility genes interacting with numerous biological and psychosocial risk factors, in addition to other influences such as developmental stage. ${ }^{1}$ However, what has evolved is a better appreciation for the complexity of the genetic contribution to mood disorders and growing interest in intermediate phenotypes related to causal pathways leading from risk to psychopathology.

\section{Genetic factors and primary mood disorders}

Family, twin and adoption studies have confirmed that major mood disorders are highly heritable, and suggest a substantial genetic contribution to treatment responsiveness as well as to tolerability (antidepressants and lithium). However, linkage and candidate-gene studies have had difficulty replicating specific genetic findings. Genome-wide association studies continue to identify a growing list of novel susceptibility genes, although overlapping samples and correction for multiple testing have complicated interpretation. ${ }^{2}$ Several meta-analyses of large cohorts have been reported and are underway, but so far genetic findings account for only a small percentage of the disease risk. Collectively, the results from genetic studies support that mood disorders are highly heterogeneous (different disease-related alleles within a gene) and result from the effects of multiple genes (polygenic).

There is increasing interest in genetic studies of treatment response and tolerability in patients with depression or bipolar disorder, which may further assist in identifying candidate risk loci associated with specific illness subtypes and provide targets for novel treatment development. Further, there is intensive

†See pp. 365-371, this issue. interest in identifying intermediate phenotypes and more homogeneous patient subgroups. An example of phenotype refinement can be seen in the use of an unequivocal response or non-response to lithium prophylaxis. An excellent response to long-term lithium monotherapy is associated with a characteristic clinical course, family history, neurobiological and genetic findings thus identifying a more genetically homogeneous bipolar subtype. ${ }^{3}$

Genetic studies are able to identify susceptibility genes associated with mood disorders; however, it remains to be worked out how genetic risk translates into illness and how each risk allele contributes to this process. Further, despite high heritability, there has long been the recognition that genetic factors do not adequately explain illness risk. A substantial body of research has shown that early adversity, ongoing difficulties and proximal undesirable life events are associated with major depressive onsets. Over the past decade there has been intensive study of genetically associated individual difference in stress sensitivity and increased vulnerability to mood disorder, with special attention being paid to the serotonin transporter linked polymorphic region (5-HTLLPR). Caspi and colleagues ${ }^{4}$ discuss the evidence supporting that in children, adolescents and young adults, the S-allele of 5-HTLLPR moderates the association between early maltreatment and subsequent depression. While acknowledging that there has been considerable recent debate about the robustness of the findings, Caspi et al conclude that the S-allele of 5-HTLLPR confers the trait of neuroticism, as well as being associated with elevated morning cortisol in adolescents at high psychosocial risk of depression. ${ }^{5}$

\section{Cortisol and primary mood disorders}

There is an emerging appreciation that epigenetic programming of gene expression may be as important as genetic polymorphisms in determining individual outcomes. Through a series of landmark animal studies, Meaney and colleagues ${ }^{6}$ have demonstrated that the early social environment can have enduring effects on stress reactivity and emotional behaviour through epigenetic mechanisms affecting the glucocorticoid receptor and hypothalamic-pituitary-adrenal axis function. These experiments have provided a model to account for developmental origins of vulnerability to disease. Distal risk factors associated with primary mood disorders in humans have included early adversity, as well as elevated cortisol. A series of studies completed by Goodyer and colleagues have demonstrated that elevations in morning salivary 
cortisol are predictive of major depression in adolescents at high risk. $^{5,7}$ The influence of the S-allele of the 5-HTLLPR on the association between morning salivary cortisol and risk of depression was recently reported in this Journal. ${ }^{5}$ The latest report in the current issue expands on previous findings, providing evidence consistent with independent moderating effects of both $B D N F$ and 5-HTLLPR genes on elevated morning salivary cortisol and the risk of major depression up to 12 months later in adolescents at high psychosocial risk.

\section{Where do we go from here?}

One important direction for future research will be to strive for replication of the current findings in an independent sample of similarly recruited high-risk adolescents. Replication of genetic findings, gene $\times$ environment interactions and genetically driven individual differences has been challenging, in part owing to different methodologies, the complexity of the genetic contribution and the heterogeneity of mood disorders. It is also important to determine whether a similar relationship between genotype, cortisol and psychopathology applies to other high-risk populations. In this regard, intermittently elevated cortisol and dexamethasone non-suppression have been reported in patients with stabilised bipolar disorder ${ }^{8}$ and in their high-risk offspring. ${ }^{9}$ Ultimately, it will be important to combine careful descriptions of the natural history of mood disorders with repeated longitudinal assessments of candidate biological and psychosocial risk factors in well-characterised subgroups of high-risk individuals.

Mapping the early clinical course starting from the premorbid state and identifying the associated pathophysiological correlates will be of critical importance to our full understanding of the evolution of illness in those at risk. Along these lines, we have reported evidence that bipolar disorder develops in a predictable sequence of clinical stages in high-risk offspring, suggesting that a developmental approach may be a helpful way to further refine phenotype definition. ${ }^{10}$ In the meantime, the most robust single predictive factor for the development of a mood disorder remains a positive family history. However, through the combination of complementary research strategies (genetic, epigenetic and highrisk), we should soon be able to identify those at greatest risk to develop end-stage illness and have an improved understanding of the intermediate causal pathways.

Anne Duffy, MD, FRCPC, Department of Psychiatry, Dalhousie University, 5850 University Avenue, Halifax, Nova Scotia B4A 1Z4, Canada. Email: anne.duffy@dal.ca First received 31 Mar 2010, final revision 4 Aug 2010, accepted 19 Aug 2010

\section{References}

1 Akiskal HS. Interaction of biologic and psychologic factors in the origin of depressive disorders. Acta Psychiatr Scand Suppl 1985; 319: 131-9.

2 McMahon FJ, Akula N, Schulze TG, Muglia P, Tozzi F, Detera-Wadleigh SD, et al. Meta-analysis of genome-wide association data identifies a risk locus for major mood disorders on 3p21.1. Nat Genet 2010; 42: 128-31.

3 Grof $\mathrm{P}$, Duffy A, Alda M, Hajek T. Lithium response across generations. Acta Psychiatr Scand 2009; 120: 378-85.

4 Caspi A, Hariri AR, Holmes A, Uher R, Moffitt TE. Genetic sensitivity to the environment: the case of the serotonin transporter gene and its implications for studying complex diseases and traits. Am J Psychiatry 2010; 167: 509-27.

5 Goodyer IM, Bacon A, Ban M, Croudace T, Herbert J. Serotonin transporter genotype, morning cortisol and subsequent depression in adolescents. Br J Psychiatry 2009; 195: 39-45.

6 Szyf M, McGowan P, Meaney MJ. The social environment and the epigenome. Environ Mol Mutagen 2008; 49: 46-60.

7 Halligan SL, Herbert J, Goodyer I, Murray L. Disturbances in morning cortisol secretion in association with maternal postnatal depression predict subsequent depressive symptomatology in adolescents. Biol Psychiatry 2007; 62: 40-6.

8 Deshauer D, Grof E, Alda M, Grof P. Patterns of DST positivity in remitted affective disorders. Biol Psychiatry 1999; 45: 1023-9.

9 Ellenbogen MA, Santo JB, Linnen AM, Walker CD, Hodgins S. High cortisol levels in the offspring of parents with bipolar disorder during two weeks of daily sampling. Bipolar Disord 2010; 12: 77-86.

10 Duffy A, Alda M, Hajek T, Sherry SB, Grof P. Early stages in the development of bipolar disorder. J Affect Disord 2010; 121: 127-35. 